\title{
Etude des écoulements en charge autour d'un obstacle par la méthode de Lattice-Boltzmann
}

\section{Study of pipe flows around an obstacle with Lattice-Boltzmann Method}

\author{
Jaouad Smily ${ }^{1}$,Bouchaib Radi ${ }^{2}$ \\ ${ }^{1}$ Laboratoire IMMII, université Hassan 1er, FST Settat,jaouadsmily@gmail.com \\ 2 Laboratoire IMMII, université Hassan 1er, FST Settat,bouchaib.radi@yahoo.fr
}

\begin{abstract}
RÉSUMÉ. La mécanique des fluides décrit des phénomènes physiques des fluides qui sont souvent gouvernés par des équations de type dérivées partielles à savoir l'équation de continuité et l'équation de Navier-Stokes . La résolution de ces équations en utilisant des méthodes classiques rencontre certaines difficultés lorsqu'il s'agit de traiter des problèmes où la géométrie du milieu est complexe ou lorsqu'on se trouve en présence de plusieurs phases d'un fluide ou de plusieurs fluides [TAO 16]. La tendance actuelle s'oriente vers une nouvelle approche pour la recherche en simulation [CFD], qui a gagné beaucoup de popularité ces dernières années, elle est appelée en anglais " Lattice Boltzmann Method " (LBM), qui est un développement relativement nouveau dans la CFD.

La méthode de Lattice-Boltzmann est une méthode de dynamique des fluides (CFD). À la place des équations de NavierStokes, l'équation discrète de Boltzmann est résolue pour simuler le comportement de fluides à l'aide d'un schéma de collision-propagation. Dans ce travail, on a intégré la LBM dans un code python afin de simuler en 2D le comportement d'un fluide en charge à l'encontre d'un obstacle, chose qui va nous servir par la suite à déterminer dans les conduites les zones les plus sollicités (vitesse maximale et pression maximale) pour mieux dimensionner à la fois la conduite et ses accessoires (vanne, clapet, etc.) et éviter leur dégradation rapide.

ABSTRACT. Fluid mechanics describes the physical phenomena of fluids which are often governed by partial derivative equations namely the continuity equation and the Navier-Stokes equation. The resolution of these equations using conventional methods encounters certain difficulties when it comes to dealing with problems where the geometry of the medium is complex or when there are several phases of a fluid or several fluids [TAO 16]. The current trend is towards a new approach to simulation research [CFD], which has gained a lot of popularity in recent years, it is called in English "Lattice Boltzmann Method" (LBM), which is a relatively new development in the Computational fluid dynamics [CFD].

The lattice Boltzmann method is a method of fluid dynamics (CFD). Instead of the Navier-Stokes equations, the Boltzmann discrete equation is solved to simulate the behavior of fluids using a collision-propagation scheme. In this work, the LBM was integrated in a python code in order to simulate in 2D the behavior of a pipe flow against an obstacle, which will be used later to determine in the pipes the most solicited zones (maximum velocity and maximum pressure) to better size both the pipe and its accessories (valve, etc.) and prevent their rapid degradation.

MOTS-CLÉS. Ecoulement en charge, Simulation, Méthode de Lattice-Boltzmann, Equation de Navier-Stokes.

KEYWORDS. Pipe Flow, Simulation, Lattice-Boltzmann Method, Navier-Stokes equation.
\end{abstract}

\section{Introduction}

Les écoulements en charge existent dans de nombreuses situations pratiques telles que les conduites industrielles, les conduites d'alimentation en eau potable, la médecine (l'écoulement du sang dans les réseaux veineux et artériels), etc. D'où réside l'importance d'étudier et simuler ce type d'écoulement pour comprendre le comportement exacte du fluide (répartition de la vitesse, pression, naissance de turbulence autour d'un obstacle, etc.).

Dans notre travail, nous avons utilisé la méthode de Lattice-Boltzmann qui est devenue une puissante alternative aux solveurs numériques conventionnels pour les équations de Navier-Stokes [PEN 11]. La méthode LBM vient combler le vide entre l'échelle microscopique et l'échelle macroscopique en se basant sur une description mésoscopique pour résoudre une fonction de distribution sur un réseau prédéfini 
[STO 13]. À l'inverse des méthodes classiques qui considèrent une description macroscopique du volume representatif élémentaire (VRE), LBM se base quant à elle sur une description mesoscopique pour résoudre une loi distibution de vitesses (distribution de Maxwell-Boltzmann) du VRE sur un réseau predéfini. De ce fait, et de point de vu numérique, l'algorithme de LBM est facilement parallélisable pour un calcul d'écoulements des fluides plus efficacement que les méthodes classiques avec maillage (éléments finis, volumes finis). La vitesse, par exemple, est calculée comme étant le moment de la fonction de distribution et par la suite pour retrouver les équations de Navier-Stokes, la LBM utilise un modèle nommé BGK développé par Bhatnagar, Gross and Krook [SAM 16].

Dans ce papier on va vous expliquer les bases de cette méthode et vous présenter les résultats obtenus lors de la simulation de l'écoulement en charge d'un fluide autour d'un obstacle.

\section{Position du problème}

Lors de son écoulement dans une conduite en charge le fluide peut rencontrer des obstacles (vanne, turbine, etc.) qui peuvent causer des changements brusques dans la répartition de la vitesse et de la pression. Notre objectif est d'étudier et simuler le comportement du fluide autour de ces obstacles et bien préciser les zones les plus sollicités pour mieux dimensionner à la fois la conduite et ses accessoires (vanne, clapet, etc.) et éviter leur dégradation rapide. Les écoulements sont décrits par l'équation de Navier-Stokes qui est une équation aux dérivées partielles non linéaires dont l'existence de solutions et la résolution constituent, dans le cas le plus général, l'un des problèmes complexes du prix du millénaire. Vue cette complexité, nous allons utiliser La méthode LBM qui est rarement utilisée dans les logiciels de simulation des fluides.

\section{Méthode de Lattice-Boltzmann (LBM)}

\subsection{Equation de Boltzmann}

L'équation de Boltzmann décrivant l'évolution spatiotemporelle d'une fonction $\mathrm{f}$ représentant la distribution des particules ayant une vitesse donnée à un endroit et à un temps donnés. Cette fonction est souvent appelée fonction de distribution et dépend donc de l'espace, de la vitesse et du temps: $f=f(x ; c ; t)$. La méthode de Lattice-Boltzmann (LBM) consiste à résoudre l'équation de Boltzmann suivante :

$$
\left.\partial_{t} f+c \nabla f+\frac{\boldsymbol{F}}{m_{p}} \frac{\partial f}{\partial \mathbf{u}}=Q(f, f)\right)
$$

où :

$\mathrm{f}$ est la fonction distribution de vitesse,

c est la vitesse d'une particule,

F représente les forces extérieures qui s'exercent sur chaque particule, mp est la masse d'une particule, $\mathrm{Q}$ est le terme de collision.

Cette équation traduit deux phénomènes : le premier est une advection de particules et le deuxième représente la collision entre les particules. 
Le terme source des équations de Navier-Stokes à modéliser est :

$$
\frac{F}{m_{p}} \frac{\partial f}{\partial \mathbf{u}}
$$

L'approximation BGK permet de modéliser le terme de collision Q comme une relaxation autour d'une distribution d'équilibre : :

$$
Q(f, f)=-\frac{1}{\tau^{\prime}}\left(f-f^{e q}\right)
$$

où $\tau^{\prime}$ est le temps de relaxation. La distribution d'équilibre est déterminée à partir de la physique statistique à un extremum d'énergie. Cette distribution est une gaussienne qui prend comme variable une vitesse $\mathrm{v}$ :

$$
f^{e q}(\mathbf{v})=\frac{\rho}{(2 \pi \theta)^{\frac{3}{2}}} e^{\left(-\frac{(\mathbf{v}-\mathbf{u})^{2}}{2 \theta}\right)}
$$

avec $\rho$ la masse volumique du fluide

et $\theta=\frac{k_{B} T}{m_{p}}$

où $k_{B}$ est la constante de Boltzmann et $\mathrm{T}$ la température. L'équation de Boltzmann devient donc :

$$
\partial_{t} f+c \nabla f+\frac{\boldsymbol{F}}{m_{p}} \frac{\partial f}{\partial \mathbf{u}}=-\frac{1}{\tau^{\prime}}\left(f-f^{e q}\right)
$$

\subsection{Modèle D2Q9}

Pour discrétiser l'équation de Boltzmann, on utilise un réseau constitué de points et de vitesses. Par exemple, pour un réseau 2D, on utilise le réseau D2Q9. Dans ce cas, "D2 "signifie que l'écoulement est à deux dimensions et "Q9" signifie qu'on a neuf directions de propagation possibles du fluide considéré.

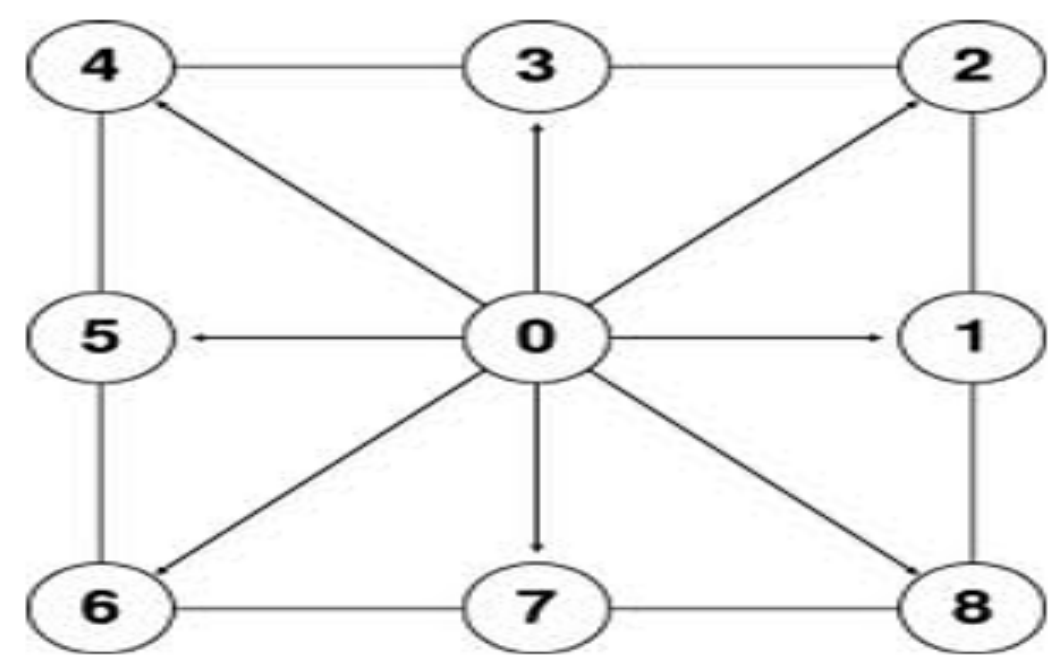

Figure 1.: Modéle D2Q9

$$
\begin{gathered}
c_{0}=(0,0) c \quad c_{1}=(1,0) c \quad c_{2}=(1,1) c \quad c_{3}=(0,1) c \quad c_{4}=(-1,1) c c_{5}=(-1,0) c \\
c_{6}=(-1,-1) c \quad c_{7}=(0,-1) c \quad c_{8}=(1,-1) c
\end{gathered}
$$


où $c=\frac{\Delta x}{\Delta t}$ est la vitesse du réseau avec $\Delta x$ le pas d'espace, $\Delta t$ le pas de temps, ces trois variables étant constantes dans une simulation.

L'équation de Boltzmann discrétisée est :

$$
f_{i}\left(x+c_{i} \Delta t, t+\Delta t\right)-f_{i}(x, t)=-\frac{1}{\tau}\left(f_{i}(x, t)-f_{i}^{e q}(x, t)\right)
$$

où $\tau=\frac{\tau^{\prime}}{\Delta t}$ et est donc sans dimension. et on a aussi :

$$
\Sigma_{i} f_{i}=\rho ; \quad \sum_{i} c_{i} f_{i}=\rho \mathrm{u}
$$

et

$$
f_{i}^{e q}=\omega_{i} \rho\left(1+3 \frac{c_{i} \cdot \mathrm{u}}{c^{2}}+\frac{9}{2} \frac{\left(c_{i} \cdot \mathrm{u}\right)^{2}}{c^{4}}-\frac{3}{2} \frac{\mathrm{u}^{2}}{c^{2}}\right)
$$

avec

$$
w_{i}= \begin{cases}\frac{4}{9} & \text { si } i=0 \\ \frac{1}{9} & \text { si } i \text { impair } \\ \frac{1}{36} & \text { si } i \text { pair }\end{cases}
$$

\section{Simulation numérique}

En se basant sur l'équation de Boltzmann discrétisée avec le modèle BGK, on a modélisé l'écoulement d'un fluide dans une conduite en charge en prenant en considération un obstacle cylindrique. Le code utilisé est écrit sous le langage PYTHON. Les données de la simulation :le nombre de Reynolds est $\operatorname{Re}=220$, le rayon du cylindre est $20 \mathrm{~m}$.

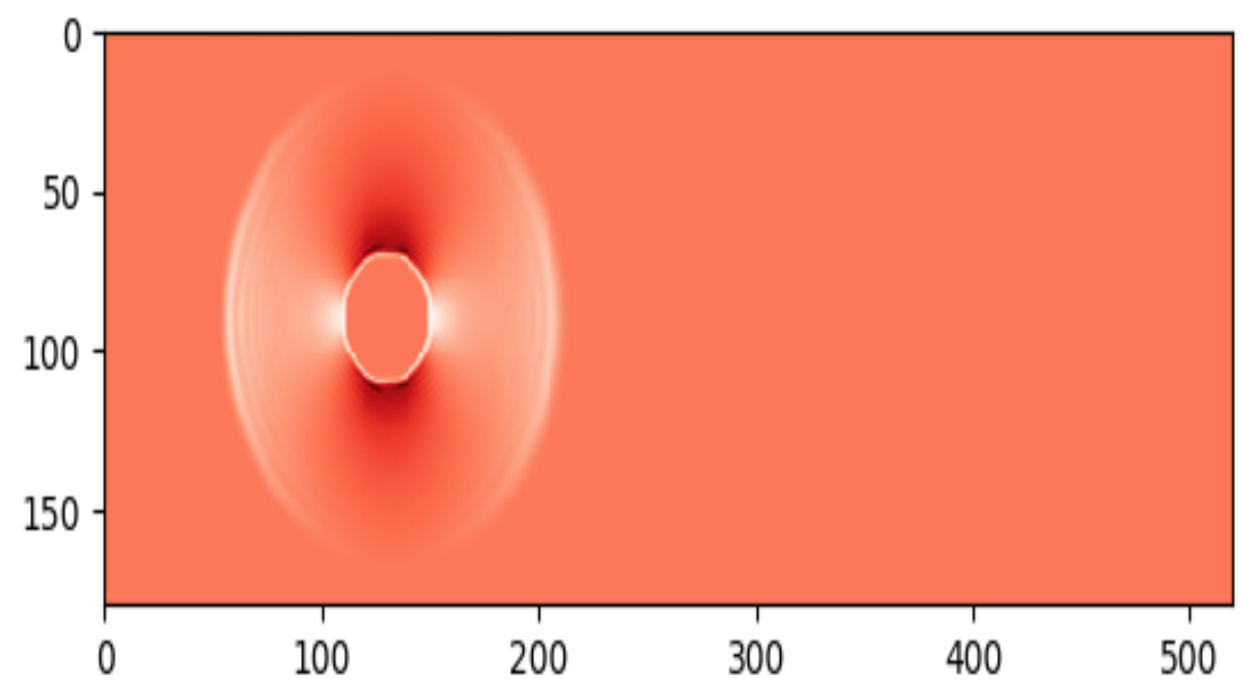

Figure 2.: Rencontre du fluide avec l'obstacle 


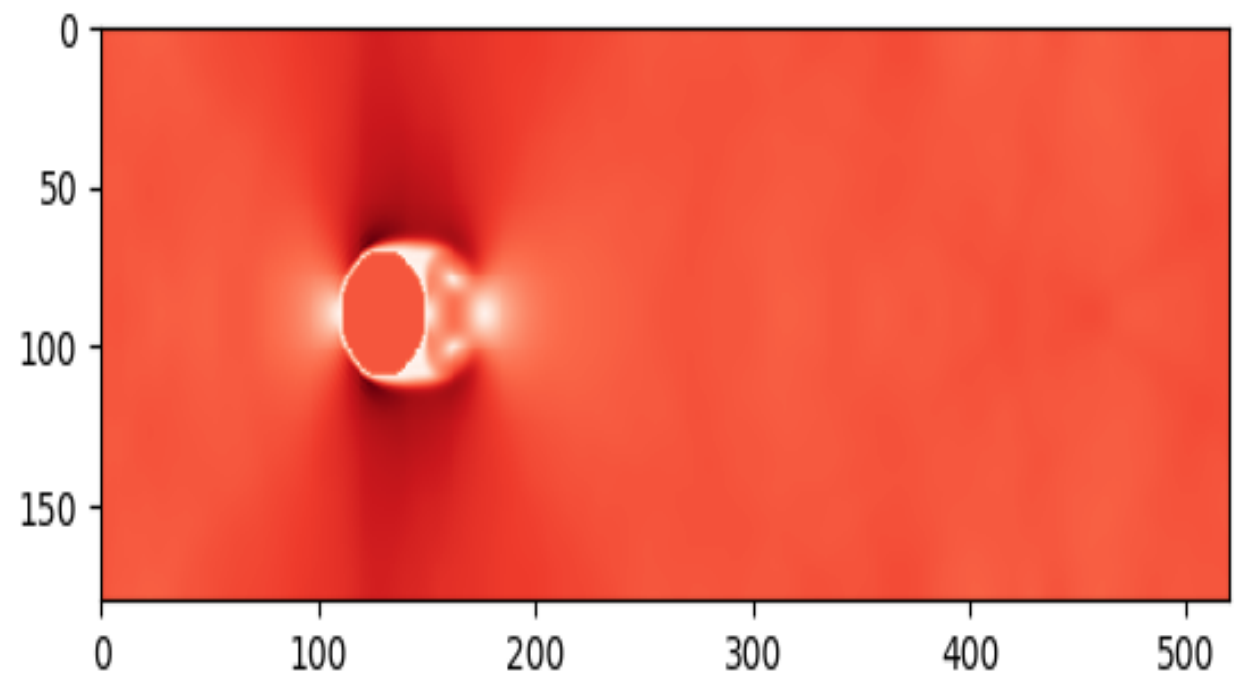

Figure 3.: Début de création du Vortex derrière l'obstacle

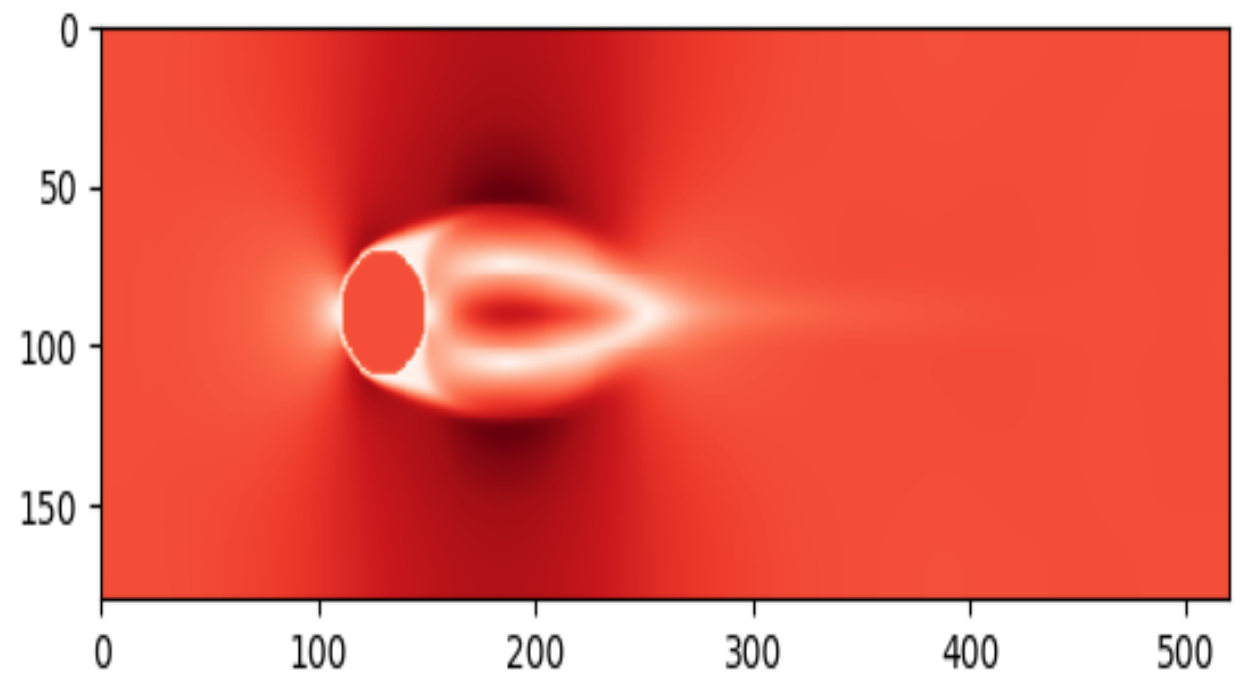

Figure 4.: Début de création du Vortex derrière l'obstacle

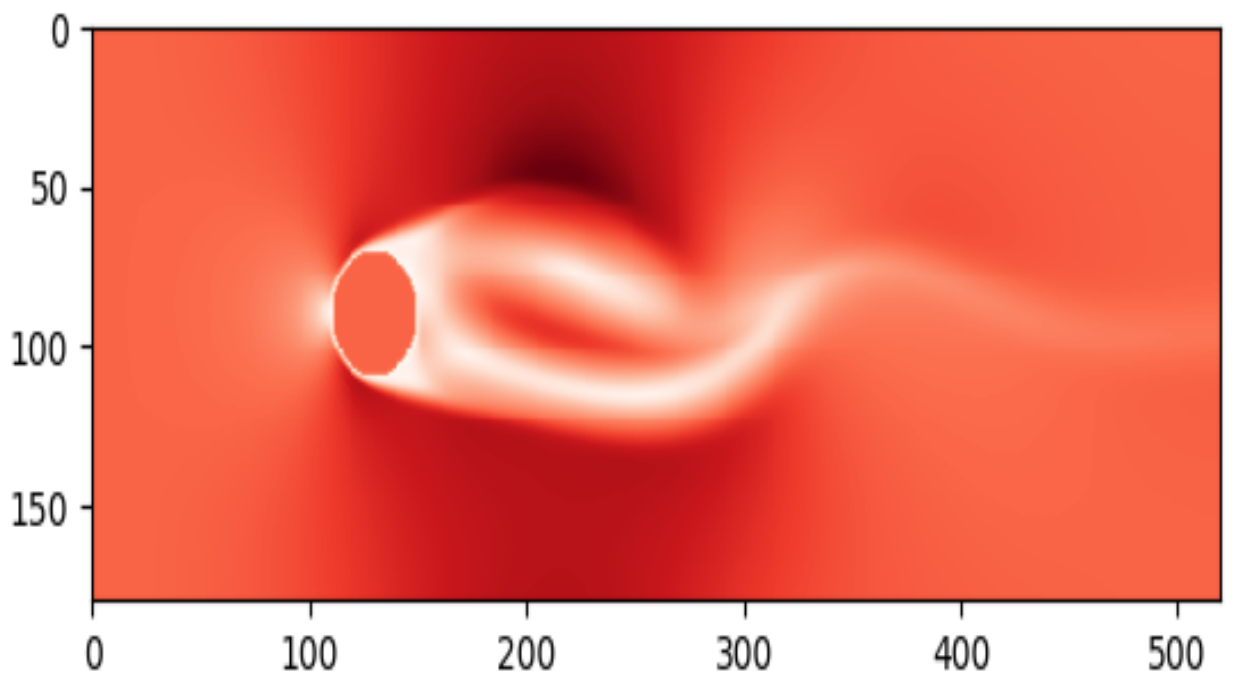

Figure 5.: Création de tourbillon derrière l'obstacle 


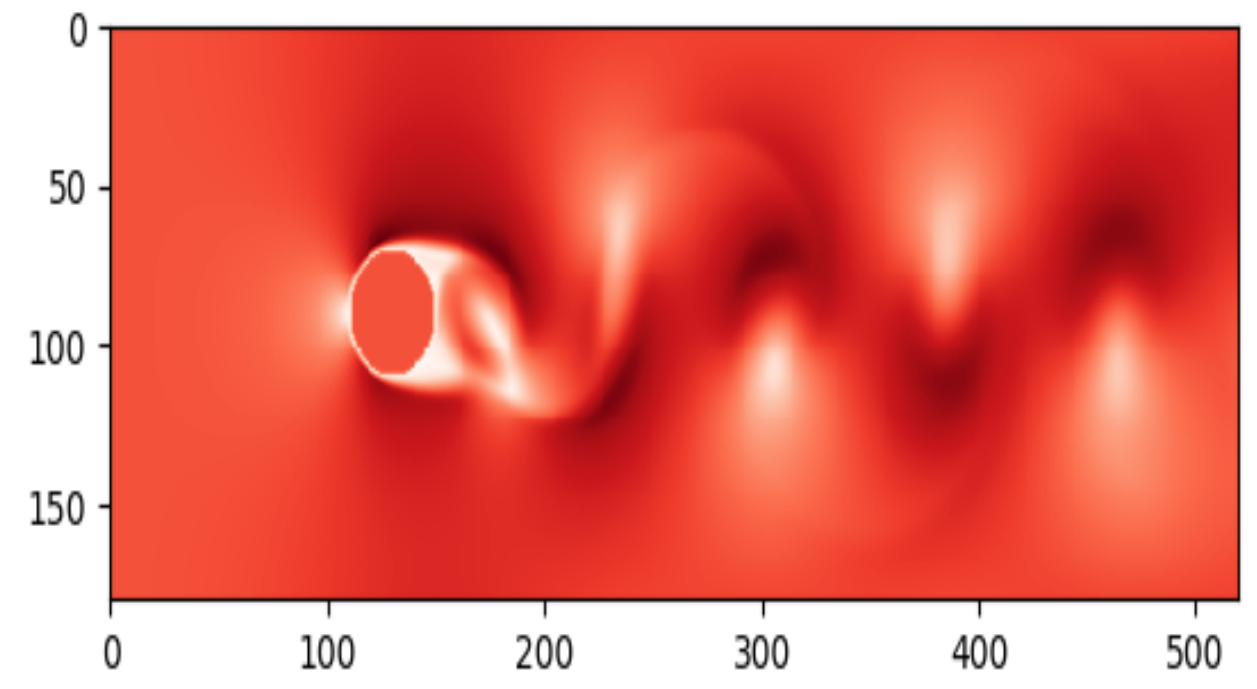

Figure 6.: Création de tourbillon derrière l'obstacle

Derrière l'obstacle, il y a une très grande perturbation de l'écoulement chose qui peut provoquer la dégradation de la conduite à long terme.

\section{Conclusion}

Rare sont les logiciels utilisant la méthode de Lattice -Boltzmann pour la simulation des écoulements. Le code développé sur python s'avère une bonne alternative pour étudier les écoulements en charge en intégrant n'importe quel obstacle à condition de bien poser les conditions aux limites. La LBM est une méthode efficace pour la simulation des fluides car on a une seule inconnue à rechercher (la fonction de distribution).

\section{Bibliographie}

SAMAOUI H., Introduction à la méthode de Boltzmann sur réseau : Application aux écoulements à surface libre. Rapport de recherche numéro : 2016.1, Université de Technologie de Compiègne (UTC), 2016.

ZHOU J.G., Lattice Boltzmann Methods for shallow water flows. Springer, New York, 2004.

PEnG C., The Lattice Boltzmann Method for Fluid Dynamics : Theory and Applications. Master of Mathematics, Ecole Polytechnique Fédérale de Lausanne, 2011.

LÉON L., Stabilité linéaire et non linéaire des schémas de Boltzmann sur réseau simulant des écoulements visqueux compressibles. Thèse de doctorat, Université Pierre et Marie Curie, 2014.

CoreIXAs C., Simulations aéroacoustiques aux grandes échelles par les méthodes Lattice- Boltzmann. Projet de fin d'étude, ISAE, 2014.

Stobiac V., Développement d'une méthode de Boltzmann sur réseau pour la simulation d'écoulements visqueux en cuves agitées. Thèse de doctorat, École Polytechnique de Montréal, 2013.

TaOussi N.,Benmamar S., Simulation numérique de l'écoulement de Poiseuille par la méthode LBM. CRND-Draria, Alger, 2016.

BEDJEGHIT E.H., Etude expérimentale et numérique d'un écoulement autour d'obstacles carré et cylindrique. Université MENTOURI Constantine, 2010. 